\title{
Hybrid Analog-Digital Precoding for mmWave Coexisting in 5G-Satellite Integrated Network
}

\author{
Deyi Peng ${ }^{1,2}$, Yun $\mathrm{Li}^{1}$, Symeon Chatzinotas ${ }^{3}$, and Bjorn Ottersten ${ }^{3}$ \\ ${ }^{1}$ Chongqing University of Post and Telecommunications (CQUPT), Chongqing 400065, China \\ ${ }^{2}$ School of Computer and Communication, Hunan Institute of Engineering(HNIE), Xiangtan 411104, China \\ ${ }^{3}$ Interdisciplinary Center for Security, Reliability and Trust (SnT), the University of Luxembourg, Luxembourg.
}

\begin{abstract}
Integrating massive multiple-input multiple-output (MIMO) into satellite network is regarded as an effective strategy to improve the spectral efficiency as well as the coverage of satellite communication. However, the inevitable intra-system and inter-system interference deteriorate the total performance of system. In this paper, we consider precoding in the 5G Satellite Integrated Network (5GSIN) with the deployment of Massive MIMO and propagation of shared millimeter-wave (mmWave) link. Taking the requirements of both frequency efficiency and energy assumption into account, a hybrid analog and digital precoding scheme in the specific scenario of 5GSIN is proposed. We model sum rate maximization problem for both of satellite and terrestrial system that incorporates maximum power constrains and minimum achievable rate requirements and formulate to a convex power allocation problem with Minimum Mean Square Error (MMSE) norm and Logarithmic Linearization method. In order to balance between performance and complexity, we propose an analog and digital separated hybrid precoding algorithm to mitigate intra-system interference. Moreover, an iterative power allocation with interference mitigation algorithm is also devised to mitigate interference from satellite to terrestrial link so that power allocation can be executed by generalized iterative algorithm. Simulation results show that our proposed hybrid precoding algorithm in 5GSIN can improve the overall spectral efficiency with a small amount of iterations.
\end{abstract}

Index Terms-5G-Satellite Integrated Network, millimeterwave, Hybrid Precoding, Power Allocation

\section{INTRODUCTION}

5G terrestrial wireless network is proved to provide high data rate with the deployment of its related innovative technologies, benefiting from its propagation quality and high frequency efficiency[1]. To improve wireless network coverage rate, satellite communication is regarded as a suitable scheme to provide services for target areas where terrestrial wireless network cannot touch[2]. Satellite combined with 5G terrestrial mobile network will provide high performance and seamless connected services as a challenging part of space information network. Meanwhile, mmWave communication with massive MIMO can improve area capacity and data rate of 5GSIN considerably[3], [4]. However, Considering the coexistence in the mmWave frequencies, interference between satellite and terrestrial link is inevitable, as well as inter-beam interference. Therefore, the performance improvement of 5GSIN depends critically on precoding scheme and transmitting power allocation method to guarantee overall system quality.

978-1-7281-4490-0/20/\$31.00 (c) 2020 IEEE
In order to mitigate the multibeam interference for multicast transmissions in satellite, a frame based precoding and multicast-aware user scheduling scheme in [5] has been proposed. In practice, payload cost and processing complexity should be reduced for forward and return link transmission few array-fed reflectors[6]. To further improve spectrum efficiency with specific energy consumption, [7] adopted Zero Forcing (ZF) and Sequential Convex Approximation (SCA) method to optimize Energy Effeciency (EE) under the total power constraint and the Quality of Service (QoS) constraints. While in [8] and [9], Imperfect Channel State Information (CSI) has been considered to manage interference in the every transmitting link. CSI estimation and feedback mechanism has also been investigated in [10] to improve performance of multibeam mobile satellite system.

Note that the aforementioned works only consider SE or EE of multibeam satellite systems, while integrating into terrestrial transmission with inter-system interference is not taken into account. To this end, authors in [11] change the phases of each beamforming weight to reduce interference for backhaul links with shared spectrum. The authors proposed a joint power and bandwidth allocation algorithm to optimize system throughput and approximate actual requirements. Efficient resource allocation technique based on multigroup precoding can improve both of system capacity and user receiving quality[12]. Therefore, [13] proposed a multilevel clustering approach and collaborative power allocation method to maximize the network capacity with guaranteeing the QoS for large number of users. Recently, in [14] and [15], cooperative multicast transmission for integrated terrestrial-satellite networks has been considered, in which beamforming vectors of terrestrialsatellite are optimized jointly to improve system capacity.

In this paper, we consider the hybrid analog digital precoding and power allocation strategy for mmWave coexisting in 5GSIN. We propose an architecture of integrated 5G and satellite wireless communication system with coexisting mmWave channel and model maximizing achievable rate with maximum power and minimum constrained QoS. In order to mitigate intra-system interference for both the terrestrial and satellite station, we propose an analog and digital separated hybrid beamforming algorithm. Then, the power allocation problem is resolved with MMSE norm and Logarithmic Linearization method. Moreover, iterative power allocation with interference mitigation algorithm is also devised to mitigate interference 
from satellite to terrestrial link so that power allocation can be executed by generalized iterative algorithm.

The rest of this paper is organized as follows. System model is presented in section II. In Section III, we formulate the power allocation problem and resolve it. And then in Section IV, performance evaluation is executed. Finally, Section V concludes the whole paper and discusses the future work.

\section{SYSTEM MODEL}

In this paper, an architecture of integrated 5G and satellite wireless communication system is proposed, as shown in Fig. 1. A Satellite and a base station (BS) provide individual services for their specific single antenna users. A BS equipped with $N_{B S}$ antennas and $N_{R F}$ RF chains communicates with $U$ terrestrial users (TUs). And similar to satellite, $M_{B S}$ antennas and $M_{R F}$ chains are assumed to provide media services for $V$ satellite users (SUs). We assume that each beam can serve only one user in this paper, so the condition $U \leq N_{R F} \leq N_{B S}$ and $V \leq M_{R F} \leq M_{B S}$ must be satisfied.

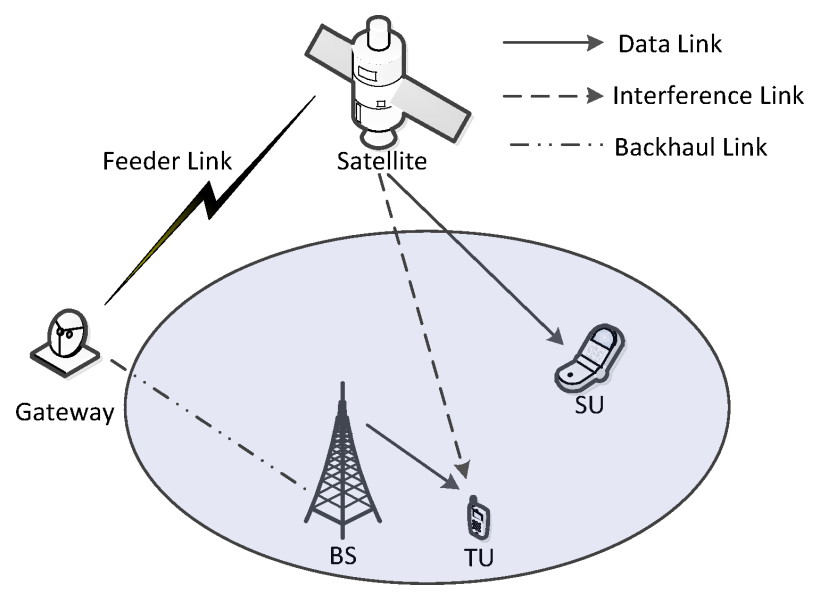

Fig. 1: The system architecture of 5GSIN.

In the practical geographical distribution, $U$ TUs locate densely confronted with inter-beam and intra-beam interference each other, while SUs hardly suffer interference from TUs[14]. Therefore, we model the receiving signal for terrestrial and satellite as the same form.

$$
\mathbf{y}=\mathbf{H x}+\mathbf{n}
$$

Where $\mathbf{y}$ is $U \times 1$ or $V \times 1$ dimension vector containing symbols for specific users. Vector $\mathbf{n}$ represents noise term of every user, which is subject to Gaussian distributed with zero mean. Regardless of traffic to be transmitted in satellite or $\mathrm{BS}$, precoding should be performed to match their wireless channel. Thus $\mathbf{x}$ in formula (1) can be represented as

$$
\mathbf{x}=\sum_{j=1}^{U \text { or } V} \mathbf{w}_{j} \mathbf{s}_{j}
$$

where $\mathbf{w}_{j}$ is the precoding vector for user $\mathbf{j}$, and $\mathbf{s}_{j}$ is the $j$ th user transmit signal, and we have $E\left[\left|s_{j}\right|^{2}\right]=1$.
Let us analyse $\mathbf{H}$ as the channel matrix based mmWave propagation. To improve spectral efficiency of the whole system, deploying mmWave in satellite system shared with terrestrial can also improve the system capacity. Therefore, the channel matrix of TU or satellite SU can be expressed as in [3],

$$
\mathbf{H}=\sqrt{\frac{N_{B S}}{L}} \sum_{l=1}^{L} \alpha^{(l)} \Phi\left(\varphi_{u}^{(l)}, \theta^{(l)}\right)
$$

Where $L$ is represented as propagated paths in mmWave link, with mean square value $E\left[\left|\alpha^{(l)}\right|^{2}\right]=\tilde{\alpha} \cdot \varphi^{(l)}$ and $\theta^{(l)}$ are respectively the azimuth and elevation Angle of Departure (AoD) of terrestrial link in the $l$ th path. Particularly, for the terrestrial link, we adopt Uniform Linear Arrays (ULAs) whose elevation angle $\theta$ can be ignored, rather than Uniform Planar Arrays (UPAs), which can be formed as

$$
\Phi_{U L A}(\varphi)=\frac{1}{\sqrt{S}}\left[1, e^{j \frac{2 \pi}{\lambda} d(\sin (\varphi)), \ldots, j(S-1) \frac{2 \pi}{\lambda} d(\sin (\varphi))}\right]^{T},
$$

where $\lambda$ denotes the signal wavelength, and $d$ is the distance of each antenna. With the above expression, We can easily obtain the ULAs of satellite link via analogy. As our analysis previously, TUs confront with obvious interference from satellite. Conversely SUs can not suffer interference from BS as the wider beam width of satellite compared with BS. Let us define $\mathbf{F}_{R F}^{S}=\left[\mathbf{f}_{R F, 1}^{S}, \mathbf{f}_{R F, 2}^{S} \cdots, \mathbf{f}_{R F, V}^{S}\right]$ for $V$ analog precoding vectors of satellite, and $\mathbf{F}_{B B}^{S}=\left[\mathbf{f}_{B B, 1}^{S}, \mathbf{f}_{B B, 2}^{S} \cdots, \mathbf{f}_{B B, V}^{S}\right]$ for $V$ digital ones. Therefore, we can derive the receiving signal

$$
y_{v}=\underbrace{\mathbf{H}_{v} \mathbf{F}_{R F}^{S} \mathbf{f}_{B B, v}^{S} \sqrt{p_{v}^{S}} \mathbf{s}_{v}}_{\text {desired signal }}+\underbrace{\sum_{i \neq v} \mathbf{H}_{v} \mathbf{F}_{R F}^{S} \mathbf{f}_{B B, i}^{S} \sqrt{p_{i}^{S}} \mathbf{s}_{i}}_{\text {intra-system interference }}+\sigma_{v}^{2}
$$

Here the data symbol $\mathbf{s}_{v}$, which transmits to $\mathrm{SU} v$, has unit power $E\left|s_{v}\right|^{2}=1$. Accordingly, the SINR can be obtained as

$$
\gamma_{v}=\frac{p_{v}^{S}\left|\mathbf{H}_{v} \mathbf{F}_{R F}^{S} \mathbf{f}_{B B, v}^{S}\right|^{2}}{\sum_{i \neq v} p_{i}^{S}\left|\mathbf{H}_{v} \mathbf{F}_{R F}^{S} \mathbf{f}_{B B, i}^{S}\right|^{2}+\sigma_{v}^{2}}
$$

Here $\sqrt{p_{v}^{S}}$ denotes the power allocated to the $v$ th SU. The first item of denominator is the interference power from the other users in the same satellite coverage, while the second item means the variance of the complex additive white Gaussian noise with the zero mean. On the other hand, analog and digital precoding is also executed at BS. Similarly, we denote analog precoding vectors as $\mathbf{F}_{R F}^{B}=\left[\mathbf{f}_{R F, 1}^{B}, \mathbf{f}_{R F, 2}^{B} \cdots, \mathbf{f}_{R F, U}^{B}\right]$, and $\mathbf{F}_{B B}^{B}=\left[\mathbf{f}_{B B, 1}^{B}, \mathbf{f}_{B B, 2}^{B} \cdots, \mathbf{f}_{B B, U}^{B}\right]$ for the digital precoding vectors. So the receiving signal for $u$ th TU can be derived to

$$
\begin{aligned}
y_{u}= & \underbrace{\mathbf{H}_{u} \mathbf{F}_{R F}^{B} \mathbf{f}_{B B, u}^{B} \sqrt{p_{u}^{B}} \mathbf{s}_{u}}_{\text {desired signal }}+\underbrace{\sum_{i \neq u} \mathbf{H}_{u} \mathbf{F}_{R F}^{B} \mathbf{f}_{B B, i}^{B} \sqrt{p_{i}^{B}} \mathbf{s}_{i}}_{\text {intra-system interference }} \\
& +\underbrace{\sum_{j} \mathbf{H}_{v} \mathbf{F}_{R F}^{S} \mathbf{f}_{B B, j}^{S} \sqrt{p_{j}^{S}} \mathbf{s}_{j}+\sigma_{u}^{2}}_{\text {inter-system interference }}
\end{aligned}
$$


Where $\mathbf{s}_{u}$ represents transmitting unit symbol with $E\left|\mathbf{s}_{u}\right|^{2}=1$. And then, its SINR of can be expressed as

$$
\gamma_{u}=\frac{p_{u}^{B}\left|\mathbf{H}_{u} \mathbf{F}_{R F}^{B} \mathbf{f}_{B B, u}^{B}\right|^{2}}{\sum_{i \neq u} p_{i}^{B}\left|\mathbf{H}_{u} \mathbf{F}_{R F}^{B} \mathbf{f}_{B B, i}^{B}\right|^{2}+\sum_{j} p_{j}^{S}\left|\mathbf{H}_{v} \mathbf{F}_{R F}^{S} \mathbf{f}_{B B, j}^{S}\right|^{2}+\sigma_{u}^{2}}
$$

where $\sqrt{p_{u}^{B}}$ is the power allocated to the $v$ th SU. The first and second items of denominator represent the interference power from the other users in the range of the same BS and satellite's interference respectively. $\sigma_{u}^{2}$ also means the variance of the complex additive white Gaussian noise with zero mean. Therefore, with $R_{v}^{S}$ and $R_{u}^{B}$ as the achievable rate of SU and TU, the sum of achievable rate of satellite and terrestrial system are

$$
\begin{aligned}
& R_{\text {sum }}^{S}=\sum_{v=1}^{V} R_{v}^{S}=\sum_{v=1}^{V} \log _{2}\left(1+\gamma_{v}\right) \\
& R_{\text {sum }}^{B}=\sum_{u=1}^{U} R_{u}^{B}=\sum_{u=1}^{U} \log _{2}\left(1+\gamma_{u}\right)
\end{aligned}
$$

Our main objective is to maximize total achievable rate of respective system with reasonable design of analog and digital precoder, as well as transmitting power. Mathematically, the combined hybrid precoding and power allocation optimization problem of satellite can be formulated as

$$
\begin{gathered}
\max _{\left\{p_{v}^{S}, \mathbf{F}_{R F}^{S}, \mathbf{F}_{B B}^{S}\right\}} R_{\text {sum }}^{S} \\
\text { s.t.C1 }: p_{v}>0, \forall v \in V \\
C 2: \sum_{v=1}^{V} p_{v}<P_{\text {max }}^{S} \\
C 3: R_{v}^{S} \geqslant R_{v}^{\text {min }}, \forall v \in V
\end{gathered}
$$

Where the optimization object $R_{\text {sum }}^{S}$, can be calculated from (9). $C 1$ is the non negative restriction of transmitting power for each SU. The condition $C 2$ is the maximum satellite system power constraint. The minimum QoS restriction with $R_{v}^{\min }$ for each SU is listed in $C 3$. Simultaneously, with the similar form and constraints, we also deduct the terrestial issue of joint hybrid precoding and corresponding power allocation as

$$
\begin{gathered}
\max _{\left\{p_{u}^{B}, \mathbf{F}_{R F}^{B}, \mathbf{F}_{B B}^{B}\right\}} R_{\text {sum }}^{B} \\
\text { s.t.C4 }: p_{u}>0, \forall u \in U \\
C 5: \sum_{u=1}^{U} p_{u}<P_{\max }^{B} \\
C 6: R_{u}^{B} \geqslant R_{u}^{\text {min }}, \forall u \in U
\end{gathered}
$$

Considering the intra-interference term in optimizing problem (11) and (15), we will focus on designing power allocation, analog precoder and digital precoder reasonably in Section IV.A and Section IV.B. And then, an interference mitigation algorithm will be proposed in Section IV.C.

\section{Problem Formulation and Solution}

Solving problem (11) and (15) directly is challenging with the non-convexity of the optimal function and constrained conditions. In this section, we decompose the intractable problem into three sub-problems. Firstly, we propose a hybrid precoding algorithm with separated analog and digital precoder. Secondly, after hybrid precoding, the original problem is transformed into a pure power allocation issue for satellite system by mitigating intra-interference. We resort to solve the convex optimization problem by MMSE norm and Logarithmic Linearization method. Lastly, with the optimal transmitting power and hybrid precoding matrix for satellite link, we use the similar iterative method to mitigate interference from satellite to terrestrial link. The particular solution will be shown in the following three subsections.

\section{A. ZF based Hybrid Precoding}

For precoding on board, processing complexity should be considered significantly. Naturally, we keep digital precoder at Gateway and analog precoder on board. Meanwhile, thanks to precoding at transmitter, physical atmosphere attenuation can be substantially reduced. Therefore, the main idea of the hybrid precoding (HP) algorithm is to maximize the transmitting power for each user and mitigate interference to other user satisfying the requirement of processing complexity. We consider analog beamforming with mmWave channel model as (3) and (4). Typically, $B$ bits quantized phase shifters have been considered to match the randomness of channel. Then, the ideal sample vectors to match mmWave channel is

$$
\theta_{u}^{\text {sample }}=\sqrt{\frac{N_{R F}}{N_{B S}}}\left\{e^{j \frac{2 \pi n}{2^{B}}}: n=0,1, \ldots, 2^{B}-1\right\}
$$

To maximize transmitting power for each user, we adopt maximum likelihood matching method as

$$
\ell_{u}=\arg \max _{n \in\left\{0,1, \ldots, 2^{B}\right\}}\left|\operatorname{angle}\left(\mathbf{H}_{u}\right)-\theta_{u}^{\text {sample }}\right|
$$

And then, with the equivalent channel matrix obtained for each user, we adopt zero-forcing (ZF) for digital precoding

$$
\mathbf{F}_{B B}=\left[\mathbf{f}_{B B, 1}, \mathbf{f}_{B B, 2}, \ldots, \mathbf{f}_{B B, U}\right]=\mathbf{H}_{e q}\left(\mathbf{H}_{e q}^{T} \mathbf{H}_{e q}\right)^{*}
$$

\section{B. MMSE based Iterative Power Allocation}

In this subsection, we will formulate and propose a iterative power allocation algorithm. By ZF based HP algorithm as previous subsection, $\mathbf{F}_{R F}$ and $\mathbf{F}_{B B}$ can be resolved for each SU or TU. Then we set $\mathbf{W}_{u, v}=\mathbf{F}_{R F} \mathbf{F}_{B B}$, so that the above optimal problem can be transformed to

$$
\begin{gathered}
\max _{\left\{p_{u(v)}\right\}} \sum_{u(v)=1}^{U(V)} \log _{2}\left(1+\frac{p_{u(v)}\left|\mathbf{H}_{u(v)} \mathbf{W}_{u(v)}\right|^{2}}{\sum_{i \neq u(v)} p_{i}\left|\mathbf{H}_{u(v)} \mathbf{W}_{i}\right|^{2}+\sigma_{u(v)}^{2}}\right) \\
\text { s.t. } C 1, C 2, C 3(C 4, C 5, C 6)
\end{gathered}
$$


Observing that (22) is still concave and hard to resolve directly, we transform it to a minimizing problem as

$$
\min _{\left\{p_{u(v)}\right\}} \sum_{u(v)=1}^{U(V)} \log _{2}\left(1+\frac{p_{u(v)}\left|\mathbf{H}_{u(v)} \mathbf{W}_{u(v)}\right|^{2}}{\sum_{i \neq u(v)} p_{i}\left|\mathbf{H}_{u(v)} \mathbf{W}_{i}\right|^{2}+\sigma_{u(v)}^{2}}\right)^{-1}
$$

$$
\text { s.t. } C 1, C 2, C 3(C 4, C 5, C 6)
$$

Then the optimal function can be written as

$$
\begin{gathered}
\eta=\log _{2}\left(1+\frac{p_{u(v)}\left|\mathbf{H}_{v} \mathbf{W}_{u(v)}\right|^{2}}{\sum_{i \neq u(v)} p_{i}\left|\mathbf{H}_{u(v)} \mathbf{W}_{i}\right|^{2}+\sigma_{u(v)}^{2}}\right)^{-1} \\
=\log _{2}\left(1-\frac{p_{u(v)}\left|\mathbf{H}_{u(v)} \mathbf{W}_{u(v)}\right|^{2}}{\sum_{\forall u(v) \in U(V)} p_{i}\left|\mathbf{H}_{u(v)} \mathbf{W}_{i}\right|^{2}+\sigma_{u(v)}^{2}}\right)
\end{gathered}
$$

On the other hand, we consider MMSE receiver with filter $\mathbf{r}_{u(v)}$ at user $u(v)$. Then the detection problem should be subject to

$$
e_{u(v)}=E\left[\left|\mathbf{s}_{u(v)}-\mathbf{r}_{u(v)} \mathbf{y}_{u(v)}\right|^{2}\right]
$$

Then, our target is to find the optimal $r_{u(v)}^{*}$ satisfying

$$
\begin{aligned}
& r_{u(v)}^{*}=\arg \min _{\left\{\mathbf{r}_{u(v)}\right\}} e_{u(v)} \\
& =\min _{\left\{\mathbf{r}_{u(v)}\right\}} E\left[\left|\mathbf{s}_{u(v)}-\mathbf{r}_{u(v)} \sum_{\forall u(v)}\right| p_{i} \mathbf{H}_{v} \mathbf{W}_{i} \mathbf{s}_{u(v)}+\left.\sigma_{u(v)}^{2}\right|^{2}\right] \\
& =\min _{\left\{\mathbf{r}_{u(v)}\right\}}\left\{1-2 \operatorname{Re}\left(\mathbf{r}_{u(v)} \sqrt{p_{u(v)}} \mathbf{H}_{u(v)} \mathbf{W}_{u(v)}\right)\right. \\
& \left.\quad+\left|\mathbf{r}_{u(v)}\right|^{2} p_{u(v)}\left|\mathbf{H}_{u(v)} \mathbf{W}_{u(v)}\right|^{2}\right\} \\
& =\left(\sqrt{p_{u(v)}} \mathbf{H}_{u(v)} \mathbf{W}_{u(v)}\right)^{T}\left(\sum_{\forall u(v)} p_{i}\left|\mathbf{H}_{u(v)} \mathbf{W}_{i}\right|^{2}+\sigma_{u(v)}^{2}\right)^{-1}
\end{aligned}
$$

And correspondingly, we can derive optimal MMSE value

$$
\begin{aligned}
& e_{u(v)}^{*}=\min _{\left\{\mathbf{r}_{u(v)}\right\}} e_{u(v)} \\
& =1-\left(p_{u(v)}\left|\mathbf{H}_{u(v)} \mathbf{W}_{u(v)}\right|\right)^{2}\left(\sum_{\forall u(v)} p_{i}\left|\mathbf{H}_{u(v)} \mathbf{W}_{i}\right|^{2}+\sigma_{u(v)}^{2}\right)^{-1}
\end{aligned}
$$

Compared (29) with (26), we have

$$
\eta=\log _{2} \min _{\left\{\mathbf{r}_{u(v)}\right\}} e_{u(v)}
$$

Remarkably, after previous derivation, the original problem has been linear. To further eliminate logarithmic calculation, we consider the following Proposition 1 from [16].

Proposition1: Let $m \in \Re^{1 \times 1}$ be a positive real value, and $f(m)=-(m n / \ln 2)+\log _{2} m+(1 / \ln 2)$. Then we have

$$
-\log (n)=\max _{m \in \Re^{1 \times 1}, m>0} f(m)
$$

where the optimal solution of $m$ is $m^{*}=1 / n$.

Assuming $m=e_{u(v)}$ and substituting $n$ with a slack variable $\xi_{u(v)}$, Combined (30) and Proposition 1, we consider

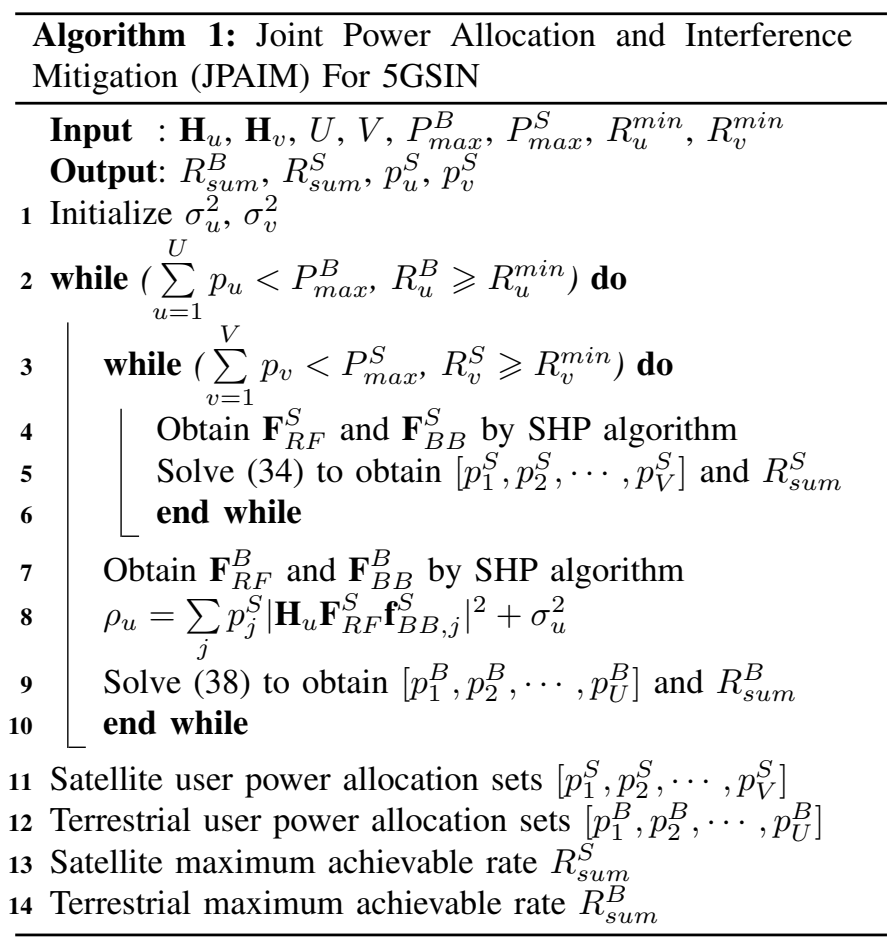

the following new optimization problem originated from (22),

$$
\begin{gathered}
\min _{\left\{p_{u(v)}\right\}} \sum_{\forall u(v) \in U(V)} \min _{\left\{\mathbf{r}_{u(v)}\right\}} \min _{\left\{\xi_{u(v)}\right\}}\left(\frac{\xi_{u(v)} e_{u(v)}}{\ln 2}-\log _{2} \xi_{u(v)}-\frac{1}{\ln 2}\right) \\
\text { s.t. } C 1, C 2, C 3(C 4, C 5, C 6)
\end{gathered}
$$

Consequently, in order to solve (32), an iterative optimization algorithm can be implemented. Specifically, problem (32) is still hard to resolve. we develop it into an iterative form as

$$
\begin{gathered}
\text { s.t. } C 1: p_{u(v)}>0, \forall u(v) \in U(V) \\
C 2: \sum_{u(v)=1}^{U(V)} p_{u(v)}^{t}<P_{\max } \\
C 3: R_{u(v)} \geqslant R_{u(v)}^{\min }, \forall u(v)
\end{gathered}
$$$$
\min _{\left\{p_{u(v)}^{t}, \xi_{u(v)}^{t}, r_{u(v)}^{t}\right\}} \sum_{\forall u(v) \in U(V)}\left(\frac{\xi_{u(v)}^{t} e_{u(v)}^{t}}{\ln 2}-\log _{2} \xi_{u(v)}^{t}-\frac{1}{\ln 2}\right)
$$

We find that (34) is transformed to a typical convex optimization problem with $e_{u(v)}^{(t)}=1-2 \operatorname{Re}\left(\mathbf{r}_{u(v)}^{(t)} \sqrt{p_{u(v)}^{(t)}} \mathbf{H}_{u(v)} \mathbf{W}_{u(v)}\right)+$ $\left|\mathbf{r}_{u(v)}^{(t)}\right|{ }^{2} p_{u(v)}^{(t)}\left|\mathbf{H}_{u(v)} \mathbf{W}_{u(v)}\right|^{2}, \quad \xi_{u(v)}^{(t)}=$ $\left[\left(\sqrt{p_{u(v)}^{(t-1)}} \mathbf{H}_{u(v)} \mathbf{W}_{u(v)}\right)^{T}\left(\sum_{\forall u(v)} p_{i}^{(t-1)}\left|\mathbf{H}_{u(v)} \mathbf{W}_{i}\right|^{2}+\sigma_{u(v)}^{2}\right)^{-1}\right]^{-1}$ and the constrained condition $C 3$ or $C 6$ can be transformed to $p_{u(v)}^{t}\left|\mathbf{H}_{u(v)} \mathbf{W}_{i}\right|^{2}-\lambda_{u(v)} \sum_{i \neq u(v)} p_{i}^{t}\left|\mathbf{H}_{u(v)} \mathbf{W}_{i}\right|^{2}-\lambda_{u(v)} \sigma_{u(v)}^{2} \geq 0$, where $\lambda_{u(v)}=2^{R_{u(v)}^{\min }}-1$. 


\section{Joint Power Allocation and Interference Mitigation}

The aforementioned generalized power allocation method is able to maximize achievable rate of unique satellite or terrestrial link. However, it is difficult to solve (15) directly for interference from satellite link. Fortunately, with the calculated hybrid precoding matrix and allocated power of satellite, intersystem interference will be mitigated to a certain extent. Therefore, we reconsider optimal problem (15) and transform it to a feasible form

$$
\begin{gathered}
\max _{\left\{p_{u}^{B}\right\}} \sum_{u=1}^{U} \log _{2}\left(1+\frac{p_{u}^{B}\left|\mathbf{H}_{u} \mathbf{W}_{u}^{B}\right|^{2}}{\sum_{i \neq u} p_{i}^{S}\left|\mathbf{H}_{u} \mathbf{W}_{i}^{B}\right|^{2}+\rho_{u}}\right) \\
\text { s.t.C4,C5,C6 }
\end{gathered}
$$

Where $\mathbf{W}_{u}^{B}$ represents hybrid matrix of terrestrial link which can be obtained by HP algorithm. $\rho_{u}$ is the combined interference and noise item, with $\rho_{u}=\sum_{j} p_{j}^{S}\left|\mathbf{H}_{u} \mathbf{F}_{R F}^{S} \mathbf{f}_{B B, j}^{S}\right|^{2}+\sigma_{u}^{2}$. Once the optimal allocated power for satellite users are determined by generalized power allocation method proposed in the previous subsection, the interference item will be mitigated. Therefore, maximizing achievable rate of terrestrial system can be implemented with the proposed generalized power allocation method. Precisely, the algorithm of joint power allocation and interference mitigation (JPAIM) for 5GSIN is given in Algorithm 1.

\section{Performance Evaluation}

In this section, simulation results based on MATLAB are provided to show the performance of our proposed algorithm in 5GSIN scenario. Considering a reference scenario of High Throughput Satellite (HTS) with one BS deployed in its coverage, we evaluate the proposed separated hybrid precoding and power allocation algorithms. Meanwhile, We assume that all of the SUs and TUs can estimate and combine their corresponding receiving signals reasonably and the Channel State Information (CSI) can be obtained perfectly for both the satellite and BS.

\section{A. Simulation Parameter Settings}

All results have been obtained by 1000 channel simulations. For our proposed iterative power allocation algorithm, we set the maximum 10 times as the iteration threshold to find the optimal maximum achievable rate. In practice, the algorithm can converge with less than 5 times iteration. With the shared frequency transmission for satellite and BS, the carrier frequency of mmWave link and $\mathrm{Ka}$ band is fixed at $30 \mathrm{GHz}[14]$, but it does not mean that our proposed 5GSIN is limited to this frequency. For satellite link transmission, specifically, we have GEO with its height $d=35786 \mathrm{~m}$. $M_{B S}=64$ antennas (or beams) with $M_{R F}=8$ employed to serve $V=8$ SUs. Propagation channel vector at Ka band can be referenced in [6]. The maximum transmitting power is restricted to $P_{\max }^{S}=60 \mathrm{dBm}$. While for terrestrial link, $U=8$ TUs have been served by $N_{B S}=64$ antennas (or beams) with $M_{R F}=8$ with mmWave channel, where $L=3$ paths included with one line-of-sight (LOS) and two non-lineof-sight (NLOS)[6]. And we set the maximum transmitting power for BS $P_{\max }^{B}=30 \mathrm{dBm}$. In order to guarantee fairness, whether satellite or terrestrial link, the minimal achievable rate is configured as $R_{\min }=0.1 * R_{\text {inf }}$, where $R_{\text {inf }}$ means the minimal achievable rate among all SUs or TUs by digital ZF precoding.

\section{B. Performance Comparisons}

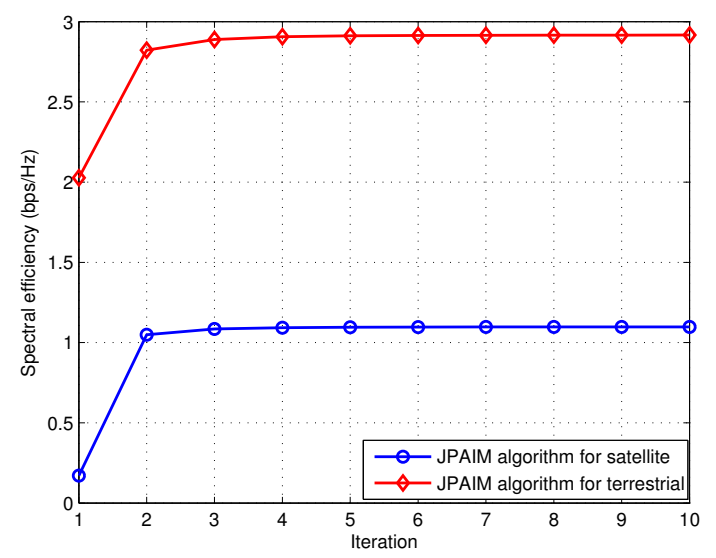

Fig. 2: Spectral efficiency versus the number of iterations.

Fig. 2 shows the convergence of our proposed JPAIM algorithm for satellite and terrestrial system respectively in algorithm at $S N R=-10 \mathrm{~dB}$. We can observe that the spectral efficiency tends to be stable after 3 or 4 times of iteration for our proposed JPAIM algorithm both in satellite and terrestrial system. In view of this, the convergence of our proposed iterative algorithm has been verified evidently. In the following simulations, the number of iteration times will be set as 5 .

In order to verify the performance of ZF based HP algorithm in subsection III.A and MMSE based Power Allocation (PA) algorithm in subsection III.B for satellite system, we compare them with equal power allocation as benchmark and full digital precoding (FDP) scheme as the upper bound in the independent satellite scenario without any terrestrial interference. As shown in Fig. 3, we can observe that our proposed MMSE based PA algorithm outperforms equal power allocation scheme. This is because that our proposed iterative power updating can efficiently mitigate the interference from other SUs. However, it is no doubt that FDP algorithm can achieve almost best spectral efficiency, as $M_{R F}$ RF chains are used to obtain multiplexing gain for all served SUs. Remarkably, MMSE based power allocation can approximate to the performance of full digital algorithm with only about $0.1 \mathrm{~dB}$ difference at the same condition, especially with high SNR. Simultaneously, in the practical 5GSIN, our proposed JPAIM is more energy efficient.

Moreover, Fig. 4 shows the performance influence of iterative interference mitigation with power allocation algorithm 
for terrestrial link with the variation of SNR in 5GSIN scenario. It can be observed that our MMSE based power allocation with HP applied in terrestrial link brings more frequency efficiency promotion than equal power allocation scheme. Meanwhile, by our proposed JPAIM algorithm, we can find that the spectral efficiency increases with the rise of SNR as intra-system and inter-system interference are mitigated efficiently. However, there is no obvious spectral efficiency promotion by JPAIM algorithm compared with MMSE base power allocation because the negative impact of inter-system interference from satellite is weak. Therefore, it is reasonable that our proposed algorithm is more suitable for the scenario of terrestrial system with robust interference from satellite.

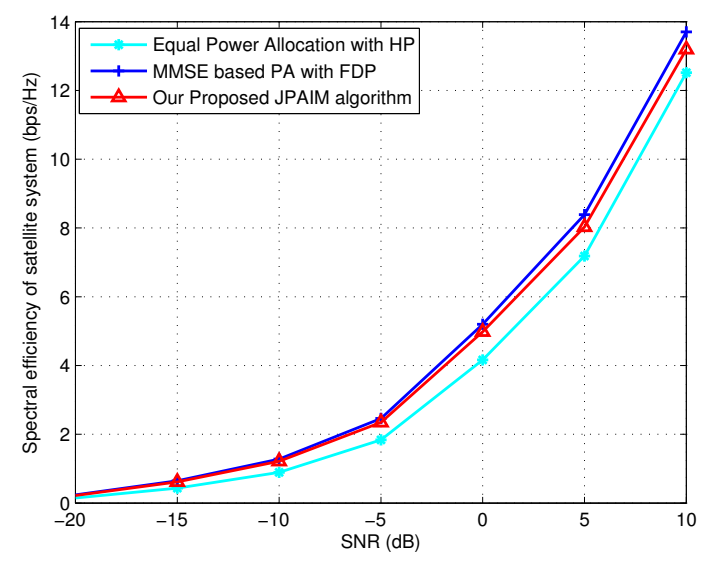

Fig. 3: Spectral efficiency versus SNR for satellite link in 5GSIN.

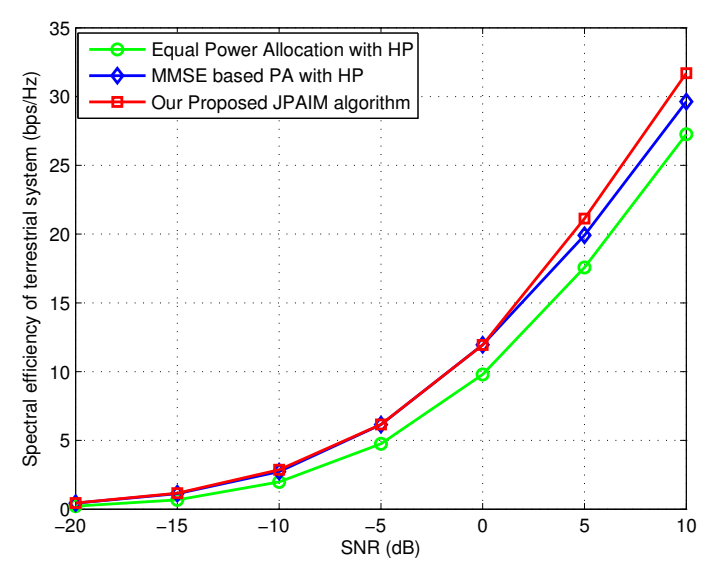

Fig. 4: Spectral efficiency versus SNR for terrestrial link in 5GSIN.

\section{CONCLusion And Future Work}

In this paper, hybrid precoding and power allocation in 5GSIN with the deployment of Massive MIMO and propagation of coexisting mmWave link have been investigated. In order to balance between performance and complexity, an hybrid precoding algorithm has been proposed to mitigate intra-system interference. Meanwhile, we also devised an iterative power allocation and interference mitigation algorithm to mitigate interference from satellite to terrestrial link so that power allocation can be executed by generalized iterative algorithm. Simulation results have been provided to verify high spectral efficiency of our proposed hybrid precoding and iterative power allocation scheme in the practical 5GSIN. In future work, we will consider cooperating and combined transceiver to further improve the performance in 5GSIN.

\section{ACKNOWLEDGMENT}

This work is supported by the Doctoral High School Talent Training Project under Grant No. BYJS201903 and the National Nature Science Foundation of China under Grant No. 61671096, partly by Chongqing Research Program of Basic Science and Frontier Technology(No. cstc2017jcyjBX0005) and Chongqing Science and Technology Innovation Leading Talent Support Program(No. CSTCCXLJRC201710).

\section{REFERENCES}

[1] J. Andrews, S. Buzzi, et al. "What will 5G be?," In IEEE J. Sel. Areas Commun., vol. 32,no. 6, pp. 1065-1082, June 2014.

[2] A. I. Prez-Neira, M. Vazquez, et al. "Signal Processing for HighThroughput Satellites Challenges in New Interference-Limited Scenarios," In IEEE Signal Processing Magazine., vol. 36, no. 4, pp. 112-131, July. 2019.

[3] C. G. Tsinos, S. Chatzinotas, et al. "Hybrid Analog-Digital Transceiver Designs for Multi-User MIMO mmWave Cognitive Radio Systems," IEEE Trans. on Cogn. Commun. and Netw., 2019.

[4] X. Liang, J Jiao, et al. "Outage analysis of multirelay multiuser hybrid satellite-terrestrial millimeter-wave networks," IEEE Wireless Communications Letters, vol. 7, no. 6, pp. 1046-16049, 2018.

[5] D. Christopoulos, S. Chatzinotas, et al. "Multicast multigroup precoding and user scheduling for frame-based satellite communi-cations," IEEE Trans. Wireless Commun., vol. 14, no. 9,pp. 4695-4707, Sept 2015.

[6] V. Joroughi, M. Vazquez, et al. "Onboard beam generation for multibeam satellite systems," IEEE Trans. Wireless Commun., vol. 16, no. 6, pp. 3714-3726, Jun. 2017.

[7] C. Qi and X. Wang. "Precoding design for energy efficiency of multibeam satellite communications," In IEEE Commun. Lett., vol. 22, no. 9,pp. 1826-1829, Sept. 2018.

[8] X, Zhang, J Wang, et al. "Robust Beamforming for Multibeam Satellite Communication in the Face of Phase Perturbations," IEEE Trans. Veh. Technol, vol. 68, no. 3, pp. 3043-3047, Mar. 2019.

[9] V, Joroughi, B Shankar, et al. "Robust Precoding and Beamforming in a Multiple Gateway Multibeam Satellite System," in Proc. IEEE Global Telecommun. Conf. (GLOBECOM), Dec. 2018, pp. 1-6.

[10] V. Joroughi, B Shankar M.R., et al. "Robust Precoding Techniques for Multibeam Mobile Satellite Systems," In in Proc. IEEE Wireless Commun. and Net. Conf. (WCNC), 2019.

[11] M. A. Vzquez, L. Blanco, et al. "Spectrum Sharing Backhaul SatelliteTerrestrial Systems via Analog Beamforming," In IEEE J. Sel. TopicsSignal Process, vol. 12, no. 2, pp. 270-281, May 2018.

[12] E, Lagunas, L Lei, et al. "Power and Flow Assignment for 5G Integrated Terrestrial-Satellite Backhaul Networks," in Proc. IEEE Wireless Commun. and Net. Conf. (WCNC), 2019.

[13] B. Y. Deng, C. X. Jiang, et al. "Joint Multigroup Precoding and Resource Allocation in Integrated Terrestrial-Satellite Networks," IEEE Trans. Veh. Technol, vol. 68, no. 8, pp. 8075-8090, Aug 2019.

[14] H. Zhang, C. Jiang, et al. "Cooperative QoS Beamforming for Multicast Transmission in Terrestrial-Satellite Networks," in Proc. IEEE Global Telecommun. Conf. (GLOBECOM), Singapore, Dec. 2017, pp. 1-6.

[15] X. Zhu, C. Jiang, et al. "Cooperative Multigroup Multicast Transmission in Integrated Terrestrial-Satellite Networks," In IEEE J. Sel. Areas Commun, vol. 36, no. 5, pp. 981-992,May 2018.

[16] Q. Zhang, Q Li, and, et al. "Robust Beamforming for Nonorthogonal Multiple-Access Systems in MISO Channels," In IEEE Trans. Veh. Technol, vol. 65, no. 12, Dec. 2016. 